\title{
Tone-on-tone binaural masking with an antiphasic masker*
}

\author{
W. A. YOST, D. W. NIELSEN, D. C. TANIS, and B. BERGERT \\ Communication Sciences Laboratory, University of Florida, Gainesville, Florida 32611
}

\begin{abstract}
One pure tone $(500 \mathrm{~Hz})$ was used to mask another pure tone of the same frequency and duration. The signal and masker were presented in three binaural stimulus configurations, Mo-So, Mo-S $\pi$, and $\mathrm{M} \pi-\mathrm{So}$. The Mo-So condition is a diotic condition; the Mo-S $\pi$ condition is a dichotic condition in which the masker is homophasic and the signal is antiphasic; and the $M \pi$-So condition is a dichotic condition in which the masker is antiphasic and the signal homophasic. The signal-to-masker ratio required for detection was measured in each condition as a function of the signal-plus-masker phase angle, $\alpha$. The data showed that the difference in detection between the Mo-S $\pi$ and $M \pi-S o$ conditions varied between $0 \mathrm{~dB}$ when $\alpha=0 \mathrm{deg}$ and $11 \mathrm{~dB}$ when $\alpha=90 \mathrm{deg}$. The difference in detection between the $\mathrm{Mo-S} \pi$ and $\mathrm{M}_{\pi}$-So conditions is due to the Os' sensitivities to the interaural phase difference present in the Mo-S $\pi$ and $\mathrm{M} \pi$-So conditions. The results are similar to those obtained in investigations involving lateralization. The difference between detection in either the Mo-S $\pi$ or $\mathrm{M} \pi$-So condition and that in the Mo-So condition (the MLD) was variable due to differences in the Os' sensitivities in the Mo-So condition.
\end{abstract}

In 1948, Hirsh observed that the detectability of a sinusoidal signal masked by a random noise was greater for certain dichotic stimulus configurations than for diotic configurations. This difference in detectability is now called the masking-level difference (MLD), and it has received considerable experimental attention (see Green \& Henning, 1966; and Green \& Yost, in press, for reviews). In the basic dichotic paradigms, a signal is added to a masker at one ear differently than it is added to the masker at the other ear.

Two of these dichotic conditions are the Mo-S $\pi$ and $\mathrm{M} \pi$-So conditions. In the Mo-S $\pi$ condition, the maskers are presented identically to both ears and the signal is added to the masker $180 \mathrm{deg}$ out of phase at one ear relative to the other ear. In the $\mathrm{M} \pi$-So condition, the masker is presented at one ear $180 \mathrm{deg}$ out of phase relative to the other ear and the signal is added to the masker identically at both ears. Shown in Fig. 1 are vector diagrams representing the addition of the signal to the masker in these two dichotic conditions as well as in the diotic condition, Mo-So (the signal and maskers presented identically to both ears).

For both the Mo-S $\pi$ and $M \pi$-So conditions, there are differences in interaural amplitude $\left(R_{R}>R_{L}\right)$ and in interaural phase $\left(\theta=\theta_{\mathbf{R}}-\theta_{\mathbf{L}}>0\right)$. These differences are not present in the Mo-So condition $\left(\mathrm{R}_{\mathrm{R}}=\mathrm{R}_{\mathrm{L}}\right.$ and $\theta$ $=0$ ). The signal-plus-masker phase angle, $\alpha$, allows one to control the interaural amplitude and interaural phase differences. In both the Mo-S $\pi$ and $\mathrm{M} \pi$-So conditions, the interaural amplitude difference decreases from a maximum at $\alpha=0 \mathrm{deg}$ to $0 \mathrm{~dB}$ at $\alpha=90 \mathrm{deg}$. The interaural phase difference increases from $0 \mathrm{deg}$ at $\alpha=$ $0 \mathrm{deg}$ to a maximum at $\alpha=90 \mathrm{deg}$. If the masker is a random noise and the signal a tone, the signal-plus masker phase angle is a random variable. Thus, one cannot control the interaural differences in experiments

*This research was supported by a National Institutes of Health training grant, an NIH Program Project grant, and an NIH special fellowship. involving noise maskers and tonal signals. Many investigators (see Yost, 1972, for a review), however, have used a tonal masker and tonal signal of the same frequency and duration in order to control $\alpha$ and to study the interaction of the interaural amplitude and phase differences in binaural masking.

In all experiments, whether or not the maskers and signals are coherent, there are differences of interaural phase and of interaural amplitude in the Mo-S $\pi$ and $\mathrm{M} \pi$-So conditions, and there are no interaural differences in the Mo-So condition. The various models of binaural masking (Durlach, 1972; Jeffress, 1972; Osman, 1971; Yost, 1971; Hafter \& Carrier, 1970) assume that in any experiment the MLD is a function of one or both of these interaural differences. However, in the Mo-S $\pi$ and $M \pi$-So conditions, the absolute values of the interaural amplitude difference and the interaural phase difference are the same. That is, the amplitudes at the right ear $\left(R_{R}\right)$ in both conditions are equal, as are the amplitudes at the left ear $\left(R_{L}\right)$. The resultant phase at the right ear $\left(\theta_{R}\right)$ has the same absolute value in both conditions as does the absolute value of the resultant phase at the left ear $\left(\theta_{\mathbf{L}}\right)$. The only difference, therefore, between the Mo-S $\pi$ and $M \pi$-So conditions is that the interaural phase difference $(\theta)$ in the $M \pi$-So condition is equal to $180 \mathrm{deg}$ minus that same phase difference $(180 \mathrm{deg}-\theta)$ in the Mo-S $\pi$ condition (see Fig. 1).

This means that in the Mo-S $\pi$ condition, the $\mathrm{O}$ is detecting the difference between a stimulus presented with no interaural differences (masker) and a stimulus presented with an interaural amplitude or interaural phase difference (signal plus masker). In the $\mathrm{M} \pi$-So condition, the absolute values of the interaural amplitude and phase differences (signal plus masker) are the same as in the Mo-S $\pi$ condition except that the discrimination is between these differences (signal plus masker) and a stimulus presented with a 180-deg interaural phase difference (masker).

Thus, any model based only on the absolute value of 


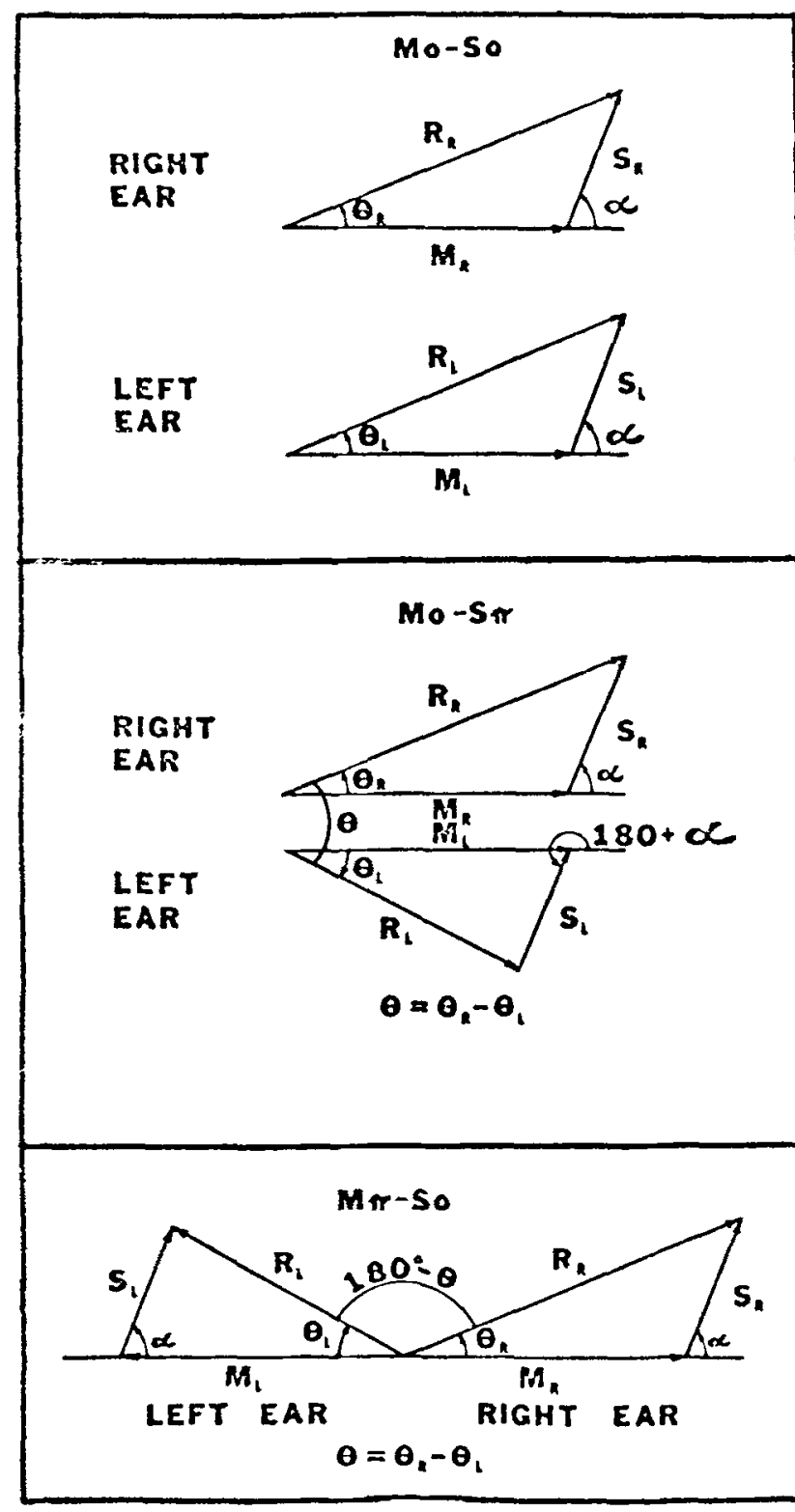

Fig. 1. The schematic diagrams representing the vectoral addition between the signal (S) and the masker $(M)$ in three interaural configurations, Mo-So, Mo-S $\pi$, and $M_{\pi}$-So. $\alpha$ is the phase angle of addition between the signal and masker, $R_{L}$ and $R_{R}$ are the resultant amplitudes at the left and right ears, respectively, and $\theta_{L}$ and $\theta_{R}$ are the resultant phase angles in each ear. The interaural phase difference is $\theta$ where $\theta=\theta_{\mathbf{R}}-\theta_{\mathbf{L}}$.

the interaural differences would predict that detection in both the Mo-S $\pi$ and $\mathrm{M} \pi$-So conditions would be the same. Many investigators (see Durlach, 1972, for a review) have shown that for a wide-band noise masker and a tonal signal there is as much as a $7-\mathrm{dB}$ difference in the signal level required for detection between the $\mathrm{Mo}-\mathrm{S} \pi$ and $\mathrm{M} \pi$-So conditions. That is, the signal is $7 \mathrm{~dB}$ easier to detect in the Mo-S $\pi$ condition than in the Mr-So condition. Therefore, the models must assume that the difference in detection is based on the 180-deg relative phase difference between the Mo-S $\pi$ and $M \pi$-So conditions.

In most models of binaural masking, a parameter can be or has been included which helps the models account for the difference in detection between the Mo-S $\pi$ and $\mathrm{M} \pi$-So conditions. However, neither the models nor the results of other experiments state explicitly which interaural difference, amplitude or phase, is primarily responsible for the difference in detection between the two conditions. It is clear that the 180 -deg relative phase difference between these two dichotic conditions must be responsible for the difference in detection. However, it is not clear which interaural difference, amplitude or phase, interacts with the relative phase difference to account for the difference in detection between the $\mathrm{Mo}-\mathrm{S} \pi$ and $\mathrm{M} \pi$-So conditions.

Therefore, it is the aim of the present study to attempt to determine which interaural difference is responsible for that difference in detection. Since a tone-on-tone binaural masking paradigm allows one to control the phase angle of addition and hence the interaural differences, a tone-on-tone masking procedure was used to investigate the difference in detection among the Mo-S $\pi, \mathrm{M} \pi$-So, and Mo-So conditions.

\section{METHOD}

All data were obtained in a two-alternative, temporal forced-choice (2ATFC) paradigm. The trial sequence consisted of a warning period, a pause, an observation interval, a pause, a second observation interval, a response interval, and a feed-back interval. The warning period was marked for the listener by a light, and feedback lights indicated to the listener which interval contained the signal plus masker. No lights marked the observation intervals (see Yost, 1970).

During each observation interval, a $350-\mathrm{m}$ sec $500-\mathrm{Hz}$ masking tone was gated with a $10-\mathrm{msec}$ rise and decay time. The masker was presented with an intensity of $70 \mathrm{~dB}$ SPL. During one of the two observation intervals, a test signal of the same frequency, duration, and rise-decay time was added to the masking tone at one of the phase angles of addition $(\alpha): 0,45$, and $90 \mathrm{deg}$. The signal plus masker was presented either in the Mo-S $\pi$ or in the $\mathrm{M} \pi$-So configuration (Fig. 1). In the Mo-So condition, only an $\alpha$ of 0 deg was used. The probability that the signal was added to the masker in the first observation interval was equal to that for the second interval. During any one block of the experiment, the $O$ was presented only one combination of phase angle of addition and interaural stimulus configuration.

Three experienced Os (D.W.N., W.A.Y., and D.C.T.) with clinically normal hearing participated in the experiment. Two levels of the test stimulus were used for each combination of interaural condition and phase angle of addition. A minimum of 300 trials was run for each stimulus combination. For each level tested, a percent correct, $P(C)$, was obtained from the equation:

$$
P(C)=[P(1 \mid I)+P(2 \mid I I)] / 2,
$$

where $P(1 \mid I)$ is the proportion of times per 100-trial block the $O$ correctly detected the signal plus masker in the first interval, and $P(2 \mid I I)$ is the proportion of correct detections in the second interval. Two points on the psychometric function were thus obtained for each stimulus combination presented in the experiment.

During training on each interaural condition, the Os were told to use the feedback lights to help them learn to make decisions. 
For the Mo-So conditions, they were instructed that their task would perhaps be to determine in which of the two intervals the tone sounded "louder." In the Mo-S $\pi$ and $\mathrm{M} \pi$-So conditions, they were told that their task could be to decide in which interval the tone sounded "off-center." In all dichotic conditions, the ear which led in phase and had the greater amplitude was the right ear.

All phase angles were measured at the electrical inputs to the TDH-39 headphones. Interaural phase relations were measured by observing the Lissajous figure produced on an oscilloscope. Phase angles of addition were calibrated by measuring voltage changes at the headphones when the signal was added to the masker.

For each $\mathrm{O}$ and for all conditions, a psychometric function of the form $d^{\prime}=m(S / M)^{k}$ (Egan et al, 1969) was fitted to the two-point psychometric function obtained from data. In the equation, $d^{\prime}$ is a criterion-free measure of the $O$ 's performance, and $m$ and $k$ are constants. The values of $m$ and $k$ leading to the best fits were used to compute the values of $20 \log \mathrm{S} / \mathrm{M}$ required for $P(C)$ s of $75 \%$.

\section{RESULTS}

The data for each of the three Os are plotted in Fig. 2 as the signal-to-masker ratio required for $75 \%$ correct detection as a function of the phase angle of addition, $\alpha$.

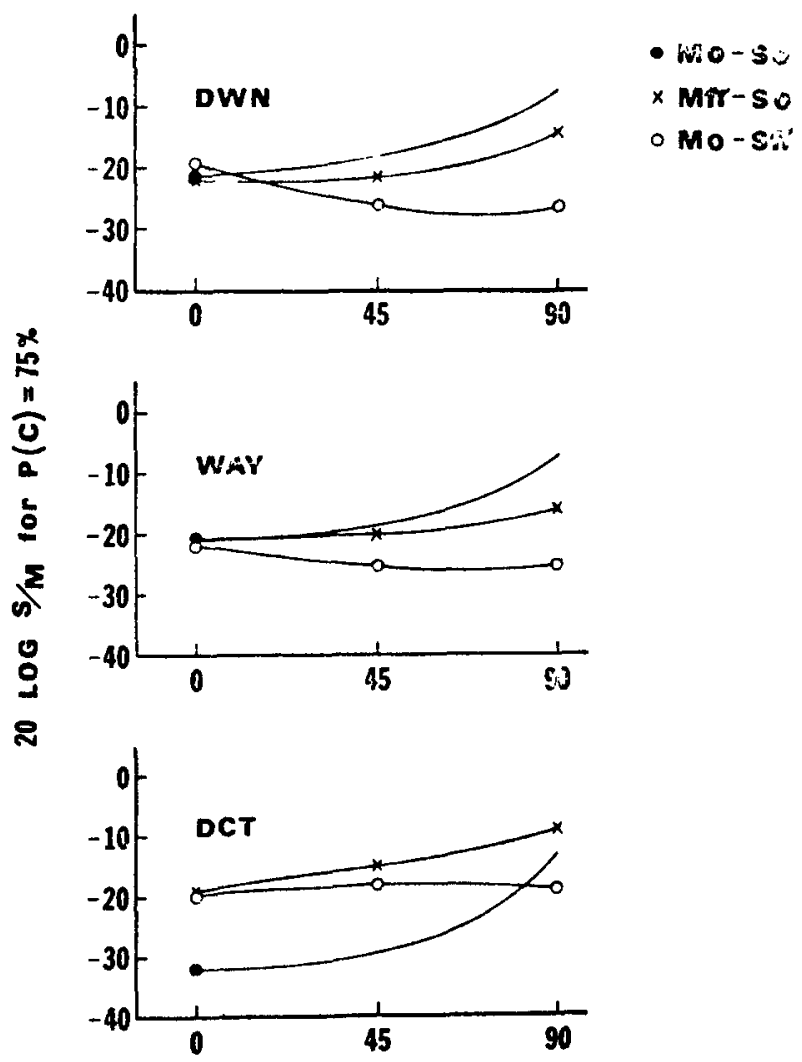

Fig. 2. The values of $20 \log \mathrm{S} / \mathrm{M}$ required for $75 \%$ correct detection plotted as a function of the signal-plus-masker phase

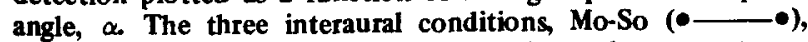
Mo-S $\pi(O-O)$, and $M_{\pi}$-So $(X-X)$, are the parameter on the curves. The three different figures are for the three $O$ s (D.W.N., W.A.Y., D.C.T.). The curve labeled Mo-So is an equal-intensity curve derived from the $\alpha=0$ deg condition (see text).

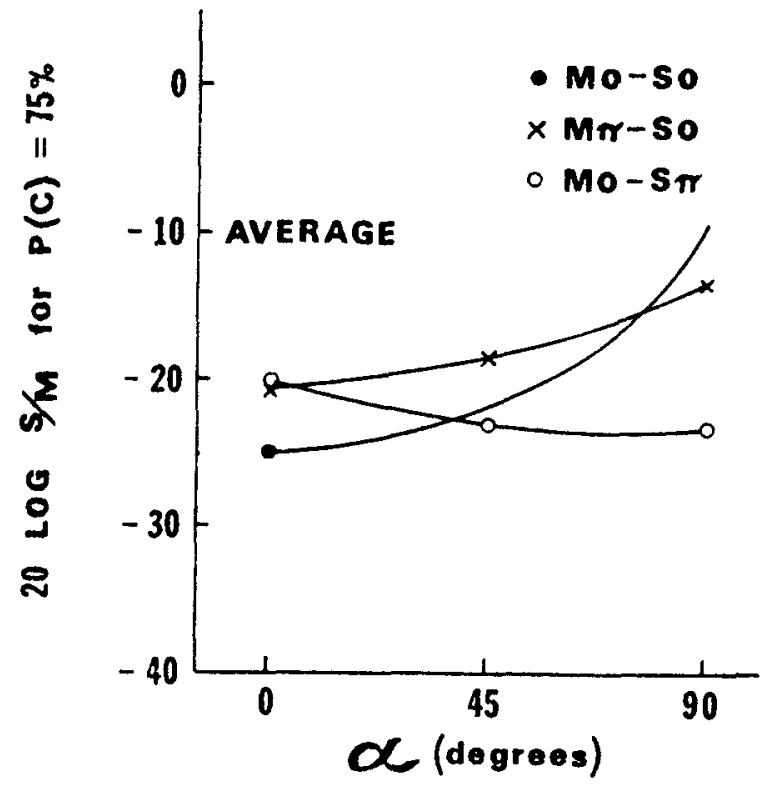

Fig. 3. The average data plotted as $20 \log S / M$ required for $75 \%$ correct detection vs the signal-plus-masker phase angle. See Fig. 2 for a description of the parameters.

The three interaural conditions, Mo-So, Mo-S $\pi$, and $\mathrm{M} \pi$-So, are represented as the parameters on the figure. The curve drawn for the Mo-So condition is derived by computing the signal-to-masker ratio as a function of $\alpha$ when the resultant signal-plus-masker amplitude is held constant at the value obtained when $\alpha=0 \mathrm{deg}$. That is, the curve represents a constant amplitude curve for the resultant amplitude as a function of $\alpha$. In the Mo-So condition, the only cue for detection is the amplitude difference between the signal-plus-masker stimulus and the masker stimulus. The signal-to-masker ratio must increase as $\alpha$ increases if this amplitude difference is to remain constant.

The average data for the three Os are shown in Fig. 3. The average values of the interaural intensive difference $\left(20 \log R_{R} / R_{L}\right)$, the interaural phase difference $(\theta=$ $\theta_{L}-\theta_{R}$ ), and the MLD (the difference in decibels between the signal-to-masker ratio obtained in either the Mo-S $\pi$ or $\mathrm{M} \pi$-So conditions and that obtained in the Mo-So condition) are shown in Table 1. These values are shown for each of the three $\alpha$ s and for the Mo-S $\pi$ and $\mathbf{M} \pi$-So conditions. No values are shown at $\alpha=0 \mathrm{deg}$ in the interaural phase row, since at $\alpha=0$ deg the interaural phase difference is always zero. No values are shown in the interaural intensity row at $\alpha=90 \mathrm{deg}$, since the interaural intensity difference is always zero at $\alpha=90 \mathrm{deg}$.

\section{DISCUSSION}

The data in Figs. 2 and 3 show that the difference in detection between the Mo-S $\pi$ and $\mathrm{M} \pi$-So conditions increases as $\alpha$ increases. Since the interaural phase 
Table 1

Average Values of the Interaural Intensive Difference $\left(20 \log R_{R} / R_{L}\right)$, the Interaural Phase Difference $\left(0=\theta_{\mathbf{L}}-\theta_{\mathbf{R}}\right)$, and the $\mathrm{MLD}^{*}$

\begin{tabular}{|c|c|c|c|c|c|c|}
\hline \multirow[b]{2}{*}{$\alpha=$} & \multicolumn{2}{|c|}{$20 \log R_{R} / R_{L}$} & \multicolumn{2}{|c|}{$\theta$} & \multicolumn{2}{|c|}{ MLD } \\
\hline & $\mathrm{Mo}-\mathrm{S} \pi$ & $\mathrm{M} \pi-\mathrm{So}$ & $\mathrm{Mo}-\mathrm{S} \pi$ & $\mathrm{M} \pi-\mathrm{So}$ & $\mathrm{Mo}-\mathrm{S} \pi$ & $\mathrm{M} \pi-\mathrm{So}$ \\
\hline $0 \mathrm{Deg}$ & $0.81 \mathrm{~dB}$ & $0.80 \mathrm{~dB}$ & - & - & $-5.0 \mathrm{~dB}$ & $-4.5 \mathrm{~dB}$ \\
\hline $45 \mathrm{Deg}$ & $0.43 \mathrm{~dB}$ & $0.73 \mathrm{~dB}$ & $6 \mathrm{Deg}$ & 10 Deg & $1.0 \mathrm{~dB}$ & $-4.0 \mathrm{~dB}$ \\
\hline $90 \mathrm{Deg}$ & - & - & $8 \mathrm{Deg}$ & $27 \mathrm{Deg}$ & $14 \mathrm{~dB}$ & $4 \mathrm{~dB}$ \\
\hline
\end{tabular}

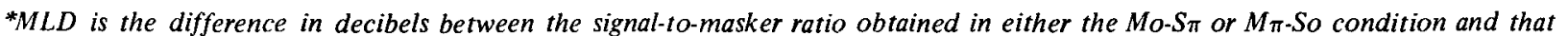
obtained in the Mo-So condition. No values are shown at $\alpha=0$ deg in the interaural phase column, since at $\alpha=0$ deg the interaural phase difference is always zero. No values are shown in the interaurai intensity column at $\alpha=90$ deg, since the interaural intensity difference is always zero at $\alpha=90 \mathrm{deg}$.

difference is also increasing as $\alpha$ increases, the data suggest that it is the interaural phase difference which is responsible for the difference in detection between the Mo-S $\pi$ and M $\pi$-So conditions.

At $\alpha=0 \mathrm{deg}$, there is only an interaural intensive difference present in both the Mo-S $\pi$ and $\mathrm{M} \pi$-So conditions, and there is no difference in detection between the two conditions. Thus, the interaural intensive difference in both conditions is the same, $0.8 \mathrm{~dB}$. At $\alpha=90 \mathrm{deg}$, there is only an interaural phase difference in both conditions. In the Mo-S $\pi$ condition, the signal-to-masker ratio required for detection was $-22 \mathrm{~dB}$, whereas in the $\mathrm{M} \pi$-So condition, it was $-11 \mathrm{~dB}$. These values yield an 8-deg interaural phase difference in the $\mathrm{Mo}-\mathrm{S} \pi$ condition and a $27-\mathrm{deg}$ interaural phase difference in the $M \pi$-So condition. Therefore, there is an $11-\mathrm{dB}$ difference in signal-to-masker ratio or a 19-deg difference in interaural phase between the Mo-S $\pi$ and $\mathrm{M} \pi$-So conditions.

These types of differences have also been observed in the lateralization experiments of Hershkowitz and Durlach (1970) and Yost (in press). In both experiments, Os were presented two stimuli in a lateral discrimination task. One stimulus, the standard, was presented with an interaural phase difference; the other stimulus, the test, was presented with this same interaural phase difference plus either an interaural intensive difference (Hershkowitz \& Durlach's study) or an additional interaural phase difference (Yost's study). The investigators could then measure the amount of interaural phase or interaural intensive difference required for detection as a function of the interaural phase difference of the standard. Both interaural differences move an image in lateral space. Thus, these studies investigated the amount of interaural phase or intensity required to move a lateral image a detectable distance provided that an image was in a particular location due to the interaural phase difference of the standard.

The stimulus conditions of the present study are identical to those used in the Hershkowitz and Durlach (1970) and the Yost (in press) investigations. That is, the masker when presented alone is the standard, and the signal plus masker is the test. The difference between the $\mathrm{Mo}-\mathrm{S} \pi$ and $\mathrm{M} \pi$-So conditions is that the interaural phase difference of the masker (standard) is changed from 0 to $180 \mathrm{deg}$. At $\alpha=0 \mathrm{deg}$, in both the $\mathrm{Mo}-\mathrm{S} \pi$ and $\mathrm{M} \pi$-So conditions, the signal-plus-masker condition is similar to the Hershkowitz and Durlach condition in that there is only an interaural intensive difference added to the interaural phase difference of the masker. In botin the present study and that of Hershkowitz and Durlach, the value of the interaural intensive difference remained at approximately $0.8 \mathrm{~dB}$, independent of the interaural phase difference of the standard (masker). At $\alpha=90 \mathrm{deg}$, in both the Mo-S $\pi$ and $\mathrm{M} \pi$-So conditions, there is a similarity between the stimulus in this study and that in Yost's experiment in that only an interaural phase difference is present. In Yost's study, the interaural phase difference of the test increased from 4 to $15 \mathrm{deg}$ as the interaural phase difference of the standard increased from 0 to $180 \mathrm{deg}$. This same 3.5 -fold increase was observed in the present study between the Mo-S $\pi$ and $\mathrm{M} \pi$-So conditions at $\alpha=90 \mathrm{deg}$. However, in this study, the Os were less sensitive to the interaural phase difference. They required $8 \mathrm{deg}$ for detection in the Mo-S $\pi$ condition and $27 \mathrm{deg}$ in the $\mathrm{M} \pi$-So condition.

Yost (in press) observed that, as the interaural phase difference of the standard increased from 0 to $180 \mathrm{deg}$, the lateral image moved from midline toward one ear. Thus, the data of the two lateralization studies and the present masking investigation suggest that as a lateral image moves toward one ear, the amount of interaural intensity required to move the image an additional detectable distance remains the same, while the interaural phase difference required for the lateral discrimination increases.

In the present study, there is a condition in which both an interaural intensive and an interaural phase difference exist when the signal plus masker is presented. At $\alpha=45 \mathrm{deg}$ in the Mo-S $\pi$ and $\mathrm{M} \pi$-So conditions, both interaural differences are present. Hafter and Carrier (1970) and Yost (1971) have proposed a lateralization model to describe the interaction of both interaural cues:

$$
\frac{\Delta \mathrm{I} \alpha}{\Delta \mathrm{Iref}}+\frac{\Delta \theta \alpha}{\Delta \theta \operatorname{ref}}=1
$$

where $\Delta \mathrm{I}$ ref is the interaural intensive reference 
condition, $\Delta \theta$ ref is the interaural phase-reference condition, and $\Delta \mathrm{I} \alpha$ and $\Delta \theta \alpha$ are the amounts of interaural phase and intensity obtained for any value of $\alpha$. The $\Delta \mathrm{I}$ ref is estimated at $\alpha=0$ deg (only an interaural intensive difference exists) and $\Delta \theta$ ref at $\alpha=90 \mathrm{deg}$ (only an interaural phase difference exists).

If the values of $\Delta \mathrm{I}$ and $\Delta \theta$ ref are estimated for the Mo-S $\pi$ condition, then at $\alpha=45 \mathrm{deg}$, Eq. 2 equals 1.1 . For the $M \pi$-So condition, when $\Delta \mathrm{l}$ ref and $\Delta \mathrm{T}$ ref are estimated, Eq. 2 equals 1.2 for the $\alpha=45 \mathrm{deg}$ condition. Thus, given that the reference conditions are estimated for both the Mo-S $\pi$ and $\mathrm{M} \pi$-So conditions, the lateralization model provides a good account of the data at $\alpha=45 \mathrm{deg}$. That is, the two interaural differences of time and phase add linearly, as in Eq. 2, independent of the interaural phase difference of the masker (referent).

The Mo-So condition enables one to estimate the amount of release from masking, or the MLD, obtained in the Mo-S $\pi$ and $\mathrm{M} \pi$-So conditions. The average values for the MLDs are shown in Table 1, but as can be seen in Fig. 2 there are significant individual differences. The variability in the MLD across Os is due primarily to the differences in detection in the Mo-So condition.

In the Mo-So condition, $\mathrm{O}$ is detecting the monaural change in intensity between the signal plus masker and the masker $(\Delta 1)$. At $\alpha=0 \mathrm{deg}$, Os D.W.N. and W.A.Y. required approximately $0.7 \mathrm{~dB}$ for $\Delta \mathrm{I}$, while D.C.T. required $0.2 \mathrm{~dB}$. Since the entire Mo-So function is derived from the data at $\alpha=0 \mathrm{deg}$, the Mo-So function of D.C.T. is quite different from D.W.N.'s or W.A.Y.'s. Such variability in the Mo-So condition has been observed by Robinson, Langford, and Yost (1974).

A change of $0.5 \mathrm{~dB}$ in $\Delta \mathrm{l}$ across 0 does not seem excessively large. However, when one computes the MLDs, one needs to obtain the signal-to-masker ratios, not $\Delta \mathrm{I}$. The $0.5-\mathrm{dB}$ difference in the $\Delta \mathrm{I}$ results in an $11-\mathrm{dB}$ difference in signal-to-masker ratio. Thus, the use of the signal-to-masker as a measure of $\Delta I$ tends to amplify the differences among $O$ s and thus the variability in the MLDs.

Although a general conclusion regarding the MLD is difficult to make in this study, the data do allow some conclusion about the difference in detection between the Mo-S $\pi$ and $M \pi$-So conditions. The difference in detection between the Mo-S $\pi$ and $M \pi$-So conditions is due primarily to the $O$ 's insensitivity to the interaural phase difference in the $\mathrm{M} \pi$-So condition.

\section{REFERENCES}

Durlach, N. I. Binaural signal detection: Equalization and cancellation theory. In J. V. Tobias (Ed.), Foundations of modern auditory theory. Vol. II. New York: Academic Press, 1972.

Egan, J. P., Lindner, W. A., \& McFadden, D. Masking-level differences and the form of the psychometric function. Perception \& Psychophysics, 1969, 6, 209-215.

Green, D. M., \& Henning, G. B. Audition. Annual Review of Psychology, 1969, 20, 105-128.

Green, D. M., \& Yost, W. A. Binaural analy sis. In W. D. Keidel and W. D. Neff (Eds.), Handbook of sensory physiology. Vol. 10. New York: Springer-Verlag, in press.

Hafter, E. R., \& Carrier, S. C. Masking level differences obtained with pulsed tonal masker. Journal of the Acoustical Society of America, 1970, 47, 1041-1048.

Hershkowitz, R. M., \& Durlach, N. I. Interaural time and amplitude jnds for a $500 \mathrm{~Hz}$ tone. Journal of the Acoustical Society of America, 1969, 46, 1464-1467.

Hirsh, I. J. The influence of interaural phase on interaural summation and inhibition. Journal of the Acoustical Society of America, 1948, 20, 536-544.

Jeffress, L. A. Binaural signal detection: Vector theory. In J. V. Tobias (Ed.), Foundations of modern auditory theory. Vol. II. New York: Academic Press, 1972.

Osman, E. A correlation model of binaural masking level differences. Journal of the Acoustical Society of America, 1971, 50, 1494-1511.

Robinson, D. E., Langford, L., \& Yost, W. A. Masking of tones by tones and noise by noise. Perception \& Psychophysics, 15, 159-167.

Yost, W. A. Tone-on-tone binaural masking. Indiana Mathematical Psychology Program Report 70-10, 1970.

Yost, W. A. Tone-on-tone masking for three binaural liste..ing conditions. Journal of the Acoustical Society of America, $1972,52,1234-1237$.

Yost, W. A. Interaural phase discriminations. Journal of the Acoustical Society of America, in press.

(Received for publication July 9, 1973; revision received September 24,1973 .) 